\title{
Exciton and electron-hole plasma formation dynamics in $\mathrm{ZnO}$
}

\author{
E. Hendry, ${ }^{1, *}$ M. Koeberg, ${ }^{2}$ and M. Bonn ${ }^{2}$ \\ ${ }^{1}$ Electromagnetic Materials Group, School of Physics, University of Exeter, Stocker Road, Exeter EX4 4QL, United Kingdom \\ ${ }^{2}$ FOM Institute for Atomic and Molecular Physics, Kruislaan 407, 1098 SJ Amsterdam, The Netherlands
}

(Received 21 November 2006; revised manuscript received 22 February 2007; published 18 July 2007)

\begin{abstract}
We employ optical pump-THz probe measurements to study the formation of excitons and electron-hole plasmas following photogeneration of a hot electron-hole gas in the direct gap semiconductor zinc oxide. Below the Mott density, we directly observe the evolution of the hot electron-hole plasma into an insulating exciton gas in the 10 to $100 \mathrm{ps}$ following photoexcitation. The temperature dependence of this process reveals that the rate determining step for exciton formation involves acoustic phonon emission. Above the Mott density, the density of the hot electron-hole plasma initially decreases very rapidly $(\sim 1.5 \mathrm{ps})$ through Auger annihilation until a stable plasma is formed close to the Mott density. In contrast to exciton formation, Auger annihilation is found to be independent of lattice temperature, occurring while the plasma is still hot.
\end{abstract}

DOI: 10.1103/PhysRevB.76.045214

PACS number(s): 71.35.Ee, 71.35.Lk, 72.20.-i, 72.20.Jv

\section{INTRODUCTION}

In semiconductors with low dielectric function, the Coulomb attraction between photoexcited electrons and holes can bind them together to form excitons rather than being separate, independent entities, as in high dielectric materials. Excitons have many interesting properties: with integer spin, excitons are promising candidates for observing BoseEinstein condensation, ${ }^{1}$ for instance. In direct gap semiconductors, the condensate of exciton polaritons has been observed. ${ }^{2}$ Excitons in direct gap semiconductors are also highly emissive, and excitonic semiconductors are widely used in electro-optic devices. In particular, zinc oxide $(\mathrm{ZnO})$ with its large exciton binding energy $(60 \mathrm{meV})$ is ideally suited for the development of polariton lasers. ${ }^{3}$

Due to the finite lifetime of excitons, however, recombination will always compete with condensation, making the knowledge of exciton formation and recombination rates essential. In addition, due to the low effective masses of electrons and holes in semiconductors, exciton binding energies are typically $\ll 1 \mathrm{eV}$, and in most semiconductors excitons only exist well below room temperature. This weak binding also leads to a Bohr radius in the $\mathrm{nm}$ range, and in the high density regime (above a critical density at which average exciton-exciton distance $r \approx a_{\text {Bohr }}$, referred to as the Mott density-see Fig. 1) screening of the electron-hole interaction reduces the boson character of excitons. Hence, one might expect exciton formation and recombination rates to be both temperature and density dependent.

Following the above-gap photoexcitation of low-dielectric semiconductors, excitons are formed in a two-step process: first the thermalization and cooling of the hot electron-hole plasma gas takes place, allowing the formation of excitons with excess center of mass momentum $(\mathbf{K} \neq 0)$. Second relaxation to the $\mathbf{K}=0$ state occurs. The majority of experimental work has employed time-resolved optical spectroscopies to probe the exciton formation dynamics (see, for example, Refs. 4-7). Unfortunately, optical absorption/emission is most sensitive to the $\mathbf{K}=0$ state of the exciton. ${ }^{8,9}$ Hence, with the exception of recent time-resolved interband luminescence ${ }^{9}$ and optical pump-terahertz $(\mathrm{THz})$ probe $^{10,11}$ measurements on exciton systems, experiments at optical frequencies generally probe the combined rates of both exciton formation and momentum relaxation. As a result, it is difficult to distinguish between a process with fast formation and slow momentum relaxation, and a process with slow formation and fast momentum relaxation.

To study the formation dynamics it is therefore advantageous to use a technique sensitive to the disappearance of free charges and the appearance of the (bound) exciton state. Due to the distinct responses of free and bound charges, ${ }^{11,12}$ $\mathrm{THz}$ time domain spectroscopy is ideally suited to follow both free and bound charge carriers on a picosecond time scale. This technique uses low energy photons to probe the intraband absorptions of the populations after photoexcitation, and can identify the different bound and unbound states through their distinct spectral responses..$^{10}$ This technique has been successfully applied to measure room temperature conductivities in $\mathrm{ZnO}$ nanorods, ${ }^{13}$ but has not yet been applied to study the mechanism of exciton formation in $\mathrm{ZnO}$ at low temperatures.

In this paper, ultrafast relaxation processes and exciton formation in $\mathrm{ZnO}$ are studied as a function of both tempera-

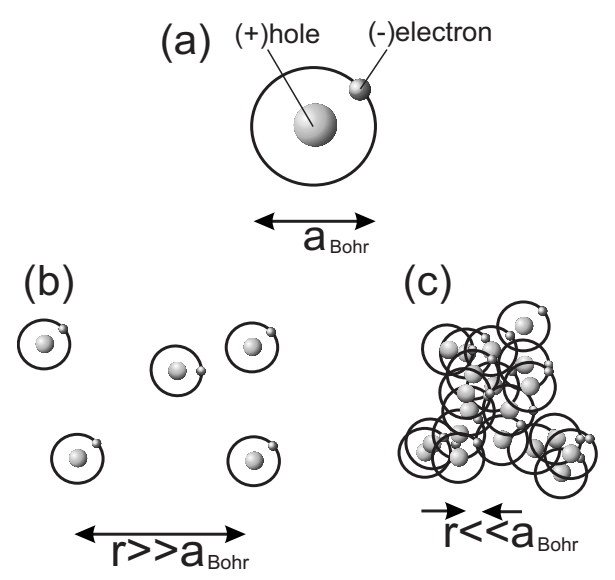

FIG. 1. (a) Wannier exciton with Bohr radius $a_{\text {Bohr }}$ (b) An exciton gas below the Mott density, with the average distance between excitations much larger than the Bohr radius of an exciton in $\mathrm{ZnO}$. (c) An exciton system excited above the Mott density. 
ture and density using optical pump- $\mathrm{THz}$ probe measurements. When the initial density of the hot electron-hole plasma is below the Mott density, we observe a slow evolution into an insulating exciton gas, on 10 to $100 \mathrm{ps}$ time scales following photoexcitation. The temperature dependence of this process reveals acoustic phonon emission as the rate determining step, suggesting that exciton formation occurs from cold charges, in contrast to previous suggestions that "hot" excitons are formed. ${ }^{7}$ Above the Mott density, we observe a very rapid $(\sim 1.5 \mathrm{ps})$ decay of the electron-hole plasma through Auger annihilation. In contrast to exciton formation, the annihilation occurs while the plasma is still hot, and is found to be independent of lattice temperature.

\section{EXPERIMENTAL PROCEDURE AND ANALYSIS}

We test two $\mathrm{ZnO}$ samples: a $12 \times 8 \times 1 \mathrm{~mm}(10 \overline{10})$-cut substrate from MaTeK (sample A), and a $5 \times 5 \times 0.5 \mathrm{~mm}$ (0001)-cut substrate from Wafer World (sample B), both made by hydrothermal growth to produce very low defect single crystals. Both are mounted in a closed cycle helium cryostat, enabling cooling down to $20 \mathrm{~K}$. The $\mathrm{ZnO}$ samples (band gap $3.37 \mathrm{eV}$ ) are photoexcited with $150 \mathrm{fs}$ laser pulses, either using $4.7 \mathrm{eV}$ photons [wavelength $\lambda=266 \mathrm{~nm}$, optical penetration depth $l_{0}=50 \mathrm{~nm}$ (Ref. 14)] or using $3.1 \mathrm{eV}$ photons $\left[\lambda=400 \mathrm{~nm}, l_{0} \approx 10 \mu \mathrm{m}\right.$ (Ref. 14)], allowing the study of dynamics in the high $\left(r \ll a_{\text {Bohr }}\right)$ and low $(r$ $\gg a_{\text {Bohr }}$ ) density regimes, respectively (see Fig. 1).

The decay dynamics of the initially excited hot plasma are investigated using optical pump-THz probe measurements. The $\mathrm{THz}$ pulses are generated by optical rectification and detected at $1 \mathrm{kHz}$ by electro-optical sampling ${ }^{15}$ using $1 \mathrm{~mm}$ thick, (110)-cut zinc telluride crystals and $800 \mathrm{~nm}, 150 \mathrm{fs}$ laser pulses $(80 \mu \mathrm{J}$ and $5 \mu \mathrm{J}$ for generation and detection, respectively). The $\mathrm{THz}$ pulses are focused onto our sample using off axis parabolic mirrors (focal length $6 \mathrm{~cm}$, numerical aperture of 0.18). The time dependent field strength of the $\mathrm{THz}$ pulses (duration $\sim 1 \mathrm{ps}$ ) transmitted through the unexcited sample $E(t)$, as well as the modulation $\Delta E(t, \tau)$ at different times $\tau$ after photoexcitation, determines the low frequency $(\nu=0.1-2 \mathrm{THz}, 0.4-8 \mathrm{meV})$ transient complex photoconductivity $\sigma(\nu, \tau)$. Since the sample can be approximated as a thin slab of excited material of thickness $l_{0}$ embedded in homogeneous material with a static dielectric function $\varepsilon \approx 8.6$, the photoconductivity is directly related to the Fourier transforms of the time domain fields ${ }^{16}$

$$
\sigma(\nu, \tau)=-\frac{2 \varepsilon_{0} c \sqrt{\varepsilon}}{l_{0}} \frac{\Delta E(\nu, \tau)}{E(\nu)},
$$

where $\varepsilon_{0}$ is the permittivity of a vacuum and $c$ is the speed of light. Since the charge distribution is inhomogeneous, $\sigma(\nu, \tau)$ represents the average frequency response of all the charges in the excitation region.

\section{RESULTS AND DISCUSSION}

\section{A. Exciton formation at low density}

Figure 2(a) shows the time evolution of the photoconductivity at $0.6 \mathrm{THz}$ for $400 \mathrm{~nm}$ excitation (fluence $\sim 0.2 \mathrm{~J} / \mathrm{m}^{2}$ )
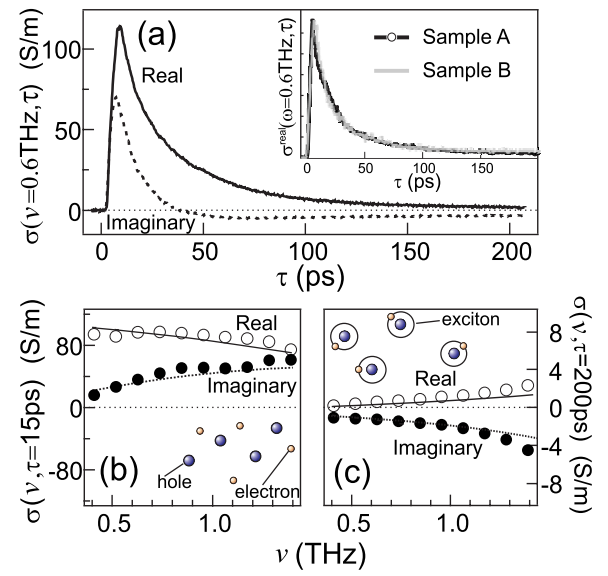

FIG. 2. (Color online) Conductivity dynamics for low density photoexcitation in $\mathrm{ZnO}$ at $30 \mathrm{~K}$. (a) The change in the complex conductivity for $0.6 \mathrm{THz}$ as a function of time after excitation (real and imaginary parts: full and dotted lines, respectively). (b) Frequency dependent complex photoconductivity measured 15 ps after excitation. The response is well described by the Drude model for free charges (lines). (c) 200 ps after excitation most of the excited electrons and holes have paired to form excitons. The increased correlation of electrons and holes is shown by the sign flip of the imaginary conductivity: the lines represent the response expected from the polarizability of the $1 \mathrm{~s}$ exciton state (Ref. 21).

at $30 \mathrm{~K}$, obtained by recording two dimensional data with $0.1 \mathrm{ps}$ steps in both $t$ and $\tau$ (by varying the time delay of detection and excitation pulses respectively), and Fourier transforming the variable $t$. At this low excitation density $\left[N_{0} \sim 2 \times 10^{22} \mathrm{~m}^{-3}\right.$ (Ref. 17)] the interexciton distance $r>30 \mathrm{~nm}$, which is considerably larger than $a_{\mathrm{Bohr}} \sim 1.8 \mathrm{~nm}$ (Ref. 18). After excitation, there is a fast rise of the signal ( $\sim 4 \mathrm{ps}$, attributed to thermalization and initial cooling of carriers), followed by a slow decay over the next 10 to $100 \mathrm{ps}$. At these low temperatures and fluences, the conductivity decays in both samples A and B are very similar [see inset of Fig. 2(a)]. This implies that the dynamics we observe in Fig. 2(a) are intrinsic to $\mathrm{ZnO}$, and unrelated to defects. Effects of anisotropy due to the different crystal cuts of sample A and samples B are also too small to observe. In the following, results are presented for sample A, measured with the $\mathrm{THz}$ polarization perpendicular to the 001 axis of the crystal.

The conductivity at $15 \mathrm{ps}$ after excitation [Fig. 2(b)] is indicative for the presence of free charges: the lines in Fig. 2(b) represent the Drude model for free charge conductivity, $\sigma(\nu, \tau)=\varepsilon_{0} \omega_{p}^{2}(\tau) /(\gamma-i 2 \pi \nu)$, with $\omega_{p}$ the density dependent plasma frequency and scattering rate $\gamma=12 \mathrm{THz}$ (a reasonable rate for a semiconducting metal oxide at low temperature ${ }^{19,20}$ ). The response is dominated by electrons, rather than holes, owing to their lower mass. ${ }^{18}$

After $\sim 50 \mathrm{ps}$ the imaginary component of the conductivity switches sign from positive to negative. This sign change originates from the disappearance of free charges and the appearance of excitons, as the restoring force introduced by the Coulomb interaction between an electron and its corresponding hole results in a shift of the response resonance from zero in the Drude model to a finite frequency $v_{n}$, and 


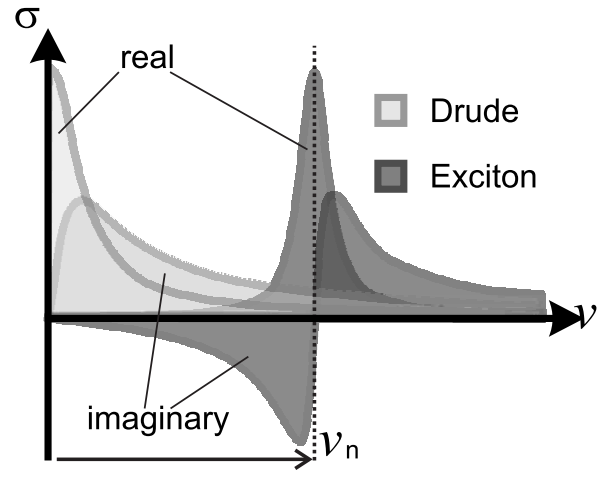

FIG. 3. Conductivity of a gas of free charges (Drude) compared to that for a gas of bound electrons and holes (exciton). The restoring force introduced by the Coulomb interaction between electrons and holes results in a shift of the response resonance from effectively zero in the Drude model to a frequency $\nu_{n}$.

resulting in a negative imaginary component of the conductivity for $\nu<\nu_{n}$ (see Fig. 3). The conductivity after 200 ps in Fig. 2(c) can be reproduced by considering the response of excitons in $\mathrm{ZnO}$ (Ref. 21) with an exciton density $N_{\text {exc }}$ $=2.6 \times 10^{22} \mathrm{~m}^{-3}$. This compares very favorably to the exciton density inferred from the excitation intensity, assuming that each absorbed photon results in the generation of an exciton, which amounts to $N_{0} \sim 2 \times 10^{22} \mathrm{~m}^{-3}$. This indicates that most of the initially excited electron-hole pairs eventually form excitons, while charge trapping and radiative exciton decay occur on longer time scales. ${ }^{22,23}$ The exciton conductivity we observe at $\tau=200 \mathrm{ps}$ persists for $\tau>1 \mathrm{~ns}$, consistent with nanosecond exciton lifetimes measured on low defect $\mathrm{ZnO}^{24}$

Since the real conductivity of free charges is considerably larger than that of the excitons, the decay of the real conductivity in Fig. 1(a) reflects the conversion of a conducting gas of free photocharges into an insulating exciton gas. This process is characterized by a single exponential decay time (gray line) with a half-life of 20 ps [Fig. 4(a)]. Increasing the $400 \mathrm{~nm}$ excitation fluence does not drastically alter the observed dynamics, confirming that the dynamics are indeed first order i.e., at these low densities the average distance in between excited pairs $(r>30 \mathrm{~nm})$ is large enough so that exciton formation by electron-hole recombination is largely geminate.

This slow evolution of a conducting gas into a gas of insulating excitons, occurring many picoseconds after photoexcitation, is similar to that observed in quantum wells using optical pump-THz probe measurements. ${ }^{11}$ The observation that this process occurs on relatively long time scales in bulk $\mathrm{ZnO}$ is remarkable given the hypothesis that the emissive exciton is formed through a "hot exciton cascade."7 In this picture, photon absorption followed by rapid emission of optical phonons by photocarriers leads to the formation of hot excitons, which subsequently cool to the emissive $(\mathbf{K}=0)$ state by slow acoustic phonon emission [Fig. 5(a)]. Since emission of optical phonons by photocarriers in semiconductors typically occurs on subpicosecond time scales, ${ }^{25}$ the slow disappearance of free charges observed here suggests that the rate determining step for exciton formation is acoustic phonon emission.
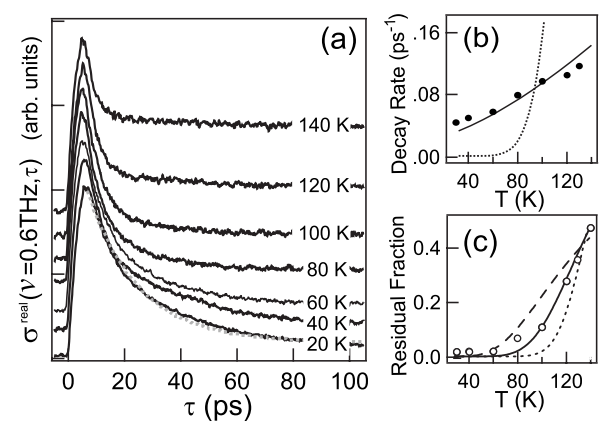

FIG. 4. (a) Temperature dependence of the exciton formation rate in sample A investigated through the decay of the real conductivity $\left(N_{0} \sim 2 \times 10^{22} \mathrm{~m}^{-3}\right.$ and normalized to peak conductivity). Traces are offset vertically for clarity. The gray dotted line is an exponential fit with time constant of 20 ps. (b) The exciton formation rate increases weakly with temperature. The dotted and full lines represent the dependence expected for formation determined by optical and acoustic phonon emission, respectively. (c) The residual dissociated exciton fraction increases with temperature. The full line indicates the dissociated fraction expected for a binding energy of $60 \mathrm{meV}$, while the dotted and dashed lines represent the dissociated fraction expected for binding energies of $30 \mathrm{meV}$ and $120 \mathrm{meV}$, respectively, calculated according to Ref. 28 .

This picture is corroborated by the temperature dependence of the exciton formation rate (again investigated through the decay of the real conductivity) shown in Fig. 4(a) $\left(N_{0} \sim 2 \times 10^{22} \mathrm{~m}^{-3}\right.$ and normalized to peak conductivity). By fitting the decaying real conductivities with exponentials, the rate of exciton formation [plotted in Fig. 4(b)] is found to increase weakly with increasing temperature (by a factor of $\sim 2.5$ from 20 to $140 \mathrm{~K}$ ). The dotted line displays the temperature dependence expected for longitudinal optical phonon scattering $\left[\propto \exp \left(-h v_{p h} / k T\right)(\operatorname{Ref} .26) v_{p h}=17 \mathrm{THz}\right]$ while the solid line represents that for acoustic phonon scattering $\left[\propto T^{3 / 2}\right.$ (Ref. 27)]: it is clear that acoustic, and not optical, phonon scattering determines the conductivity decay

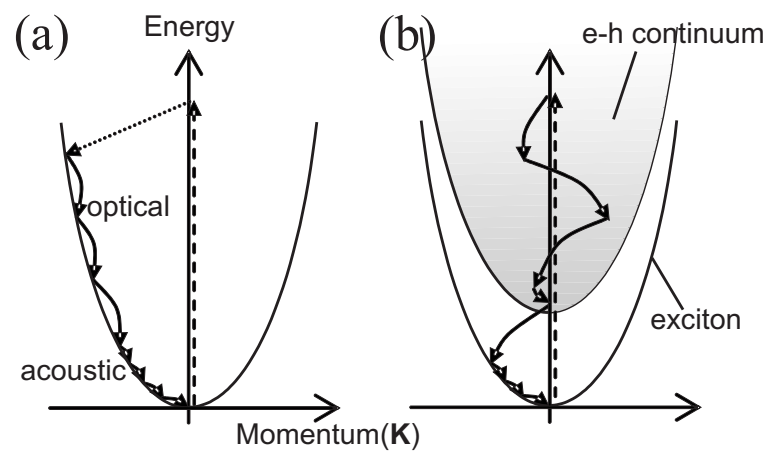

FIG. 5. (a) Exciton formation illustrated in terms of the "hot exciton cascade": after excitation, hot exciton cooling occurs by optical (large curved arrows) and, as the exciton falls to the bottom of the well, acoustic (small curved arrows) phonon emission. (b) $\mathrm{THz}$ measurements suggest that the motion of electrons and holes remains uncorrelated for much of the cooling process. In this case, a more realistic description involves cooling through the electronhole continuum, where the uncorrelated electron and holes have, on average, no net center of mass momentum $(\mathbf{K})$. 
i.e., most cooling occurs through the electron-hole continuum [see Fig. 5(b)], and the final step before exciton formation involves acoustic phonons emitted by free carriers.

For higher temperatures, the long time equilibrium free charge density (the measured plateau after the initial plasma decay) increases due to thermal dissociation of excitons. The temperature dependence of the residual dissociated fraction (defined as the magnitude of the plateau conductivity normalized by that at the peak) is displayed in Fig. 4(c). The solid line shows the dissociation fraction expected for a simple two component equilibrium (bound and free states) with the forward step having an activation barrier $\sim 60 \mathrm{meV}$ (the exciton binding energy). ${ }^{28}$

\section{B. Plasma formation at high density}

Photoexcitation at $266 \mathrm{~nm}$ results in a significantly higher excitation density, resulting in an average interexciton distance $r<3 \mathrm{~nm} \approx 2 a_{\text {Bohr }} .{ }^{18}$ In this regime the photoconductivity is observed to be frequency independent over our probe spectrum, as expected for a high density electron-hole gas. ${ }^{29}$ The temporal evolution of the conductivity at $0.6 \mathrm{THz}$ for excitation fluence $\sim 1.5 \mathrm{~J} / \mathrm{m}^{2}\left[N_{0} \sim 2 \times 10^{25} \mathrm{~m}^{-3}\right.$ (Ref. 17)], is shown in Fig. 6(a). While the imaginary conductivity is essentially zero at all pump-probe times, the real conductivity rapidly decays with an initial half-life $\tau_{1 / 2} \sim 1.5 \mathrm{ps}$, i.e. 1-2 orders of magnitude faster than exciton formation.

Since the photomodulation of the $\mathrm{THz}$ pulse is essentially proportional to the product of density and penetration depth [see Eq. (1)], we rule out plasma expansion as the cause of the decay in Fig. 6. Instead, the fast decay is attributed to many body (Auger) annihilation of charges, known to be fast for other exciton systems, ${ }^{30}$ and corroborated by the higher order (i.e. nonexponential) dynamics in Fig. 6. Auger recombination ${ }^{30}$ is mediated by enhanced charge-charge interactions at high density and involves collisions between (at least) three charges to redistribute the energy given out when an electron and a hole recombine. Auger recombination times are determined by the Auger constant $C_{A}$ (Ref. 30) through $\tau_{1 / 2}=\ln (2) /\left(C_{A} N_{0}{ }^{2}\right)$. Though the dynamics here are complicated by several experimental issues,${ }^{31}$ for the real conductivity decay in Fig. 6(a) we estimate from $\tau_{1 / 2}$ that $C_{A} \approx 1 \times 10^{-39} \mathrm{~m}^{6} / \mathrm{s}$ for $\mathrm{ZnO}$. This is around four orders of magnitude larger than for indirect semiconductors such as Silicon $\left[C_{A} \approx 3 \times 10^{-43} \mathrm{~m}^{6} / \mathrm{s}\right.$ (Ref. 32)], where Auger recombination is phonon assisted due to the mismatch in electron and hole momenta. In contrast, the broad emission under high density excitation ${ }^{33}$ suggests that recombination in $\mathrm{ZnO}$ is radiative.

After the fast initial decrease, the decay of conductivity clearly slows for times $>20 \mathrm{ps}$. Ignoring charge diffusion in between pump and probe pulses (which we estimate to be a minor effect ${ }^{29}$ ), and comparing to the peak conductivity, we estimate that the plasma reaches a plateau density $\sim 2$ $\times 10^{24} \mathrm{~m}^{-3}$. This more stable density is only weakly dependent on the initial excitation intensity [the decay for a fivefold increase in fluence is plotted in Fig. 6(b)]. For our estimate of $C_{A} \approx 1 \times 10^{-39} \mathrm{~m}^{6} / \mathrm{s}$, Auger recombination rates fall quickly with decreasing density, and become smaller than

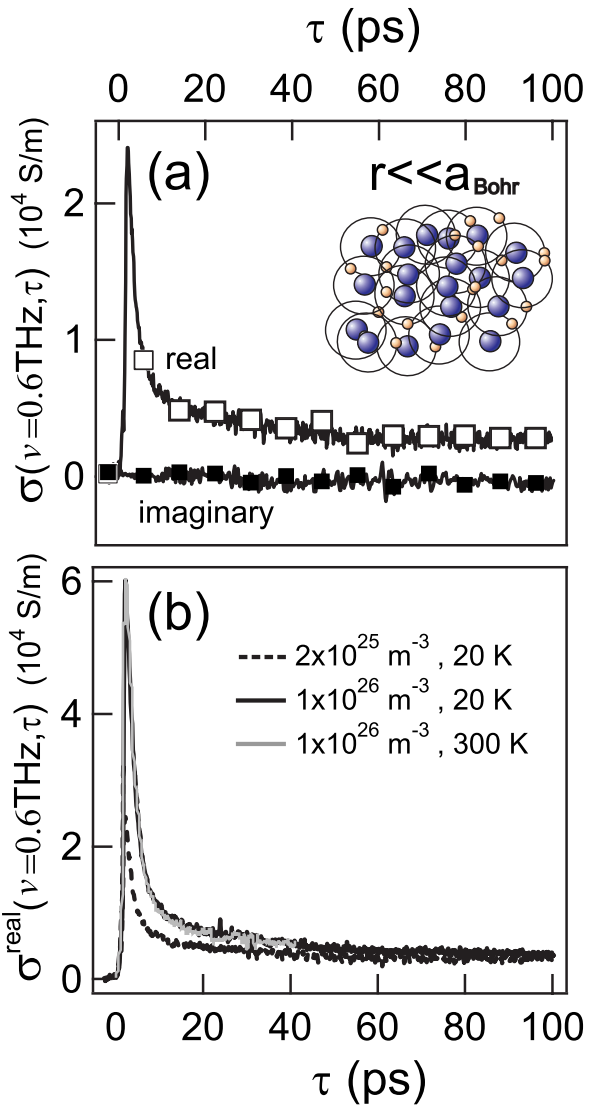

FIG. 6. (Color online) Conductivity dynamics for high excitation densities in sample A. At such high densities $\left(r \ll a_{\text {Bohr }}\right)$, the system can no longer be described as a gas of excitons. (a) The open (real) and closed (imaginary) squares show the nonexponential dynamics for $266 \mathrm{~nm}$ photon excitation resulting in $N_{0} \sim 2$ $\times 10^{25} \mathrm{~m}^{-3}$. (b) Decay of the real component for two different excitation densities: while both measurements show similar fast decays, the magnitude of the signal on long times does not vary strongly with excitation density. The dynamics are temperature independent: the gray line represents a measurement at $300 \mathrm{~K}$, coinciding perfectly with the $20 \mathrm{~K}$ data.

exciton radiative recombination rates ${ }^{24}$ for a density $<2-3$ $\times 10^{24} \mathrm{~m}^{-3}$. This corresponds to $r \approx 7 \mathrm{~nm}$, the same order of magnitude as the Bohr radius $a_{\text {Bohr }}$ (Ref. 18) i.e.; the plasma is stabilized when the distance in between electron-hole pairs is comparable to the exciton Bohr radius.

In contrast to the low density regime (i.e., $r \gg a_{\text {Bohr }}$, where bound insulating excitons are formed), it is notable from the residual real conductivity that, for $r \approx a_{\text {Bohr }}$, the plasma remains in a conducting state. This conducting state persists on nanosecond time scales. A similar insulator-to-conductor transition on increasing the electron-hole density has also been observed with optical pump-THz probe measurements in quantum well structures. ${ }^{34}$

In indirect semiconductors, since the process is phonon mediated, annihilation has a strong temperature dependence. ${ }^{32}$ For $\mathrm{ZnO}$, the high density plasma decay in Fig. 6(b) is essentially independent of lattice temperature. Furthermore, the decay due to annihilation is faster than cooling of carriers in the low density regime [given by the rise of the 
signal in Fig. 2(a)], suggesting that annihilation takes place directly from the hot plasma, before cooling. Such ultrafastfast depopulation of the conduction band is destructive toward many electro-optical applications of $\mathrm{ZnO}$ in the high density regime, specifically to lasing in $\mathrm{ZnO} .{ }^{35}$

\section{SUMMARY}

In conclusion, we have measured the ultrafast photoconductivity of $\mathrm{ZnO}$ using optical pump- $\mathrm{THz}$ probe measurements. Below the Mott density, we observe slow evolution of free charges to excitons in the 10 to 100 ps following photoexcitation, indicating that cooling is almost complete before exciton formation occurs. Above the Mott density, the initial high density electron-hole plasma decays very rapidly $(\sim 1.5 \mathrm{ps})$ through Auger annihilation. In contrast to exciton formation, annihilation is found to be independent of lattice temperature, occurring while the plasma is still hot.

\section{ACKNOWLEDGMENTS}

This work is part of the research program of the Stichting voor Fundamenteel Onderzoek der Materie (FOM), which is financially supported by the Nederlandse Organisatie voor Wetenschappelijk Onderzoek (NWO).
*Email address: E.Hendry@exeter.ac.uk

${ }^{1}$ I. E. Perakis, Nature (London) 417, 33 (2002).

${ }^{2}$ H. Deng, G. Weihs, C. Santori, J. Bloch, and Y. Yamamoto, Science 298, 199 (2002).

${ }^{3}$ M. Zamfirescu, A. Kavokin, B. Gil, G. Malpuech, and M. Kaliteevski, Phys. Rev. B 65, 161205(R) (2002).

${ }^{4}$ P. W. M. Blom, P. J. van Hall, C. Smit, J. P. Cuypers, and J. H. Wolter, Phys. Rev. Lett. 71, 3878 (1993).

${ }^{5}$ T. C. Damen, J. Shah, D. Y. Oberli, D. S. Chemla, J. E. Cunningham, and J. M. Kuo, Phys. Rev. B 42, 7434 (1990).

${ }^{6}$ H. Kalt, J. Hoffmann, M. Umlauff, W. Langbein, and J. M. Hvam, Phys. Status Solidi B 206, 103 (1998).

${ }^{7}$ D. Kovalev, B. Averboukh, D. Volm, B. K. Meyer, H. Amano, and I. Akasaki, Phys. Rev. B 54, 2518 (1996).

${ }^{8}$ D. S. Chemla and J. Shah, Nature (London) 411, 549 (2001).

${ }^{9}$ J. Szczytko, L. Kappei, J. Berney, F. Morier-Genoud, M. T. Portella-Oberli, and B. Deveaud, Phys. Rev. Lett. 93, 137401 (2004).

${ }^{10}$ S. W. Koch, M. Kira, G. Khitrova, and H. M. Gibbs, Nat. Mater. 5, 523 (2006).

${ }^{11}$ R. A. Kaindl, M. A. Carnahan, D. Hagele, R. Lovenich, and D. S. Chemla, Nature (London) 423, 734 (2003).

${ }^{12}$ E. Hendry, J. M. Schins, L. P. Candeias, L. D. A. Siebbeles, and M. Bonn, Phys. Rev. Lett. 92, 196601 (2004).

${ }^{13}$ J. B. Baxter and C. A. Schmuttenmaer, J. Phys. Chem. B 110, 25229 (2006).

${ }^{14}$ For $400 \mathrm{~nm}$ pump pulses, we expect absorption to occur through a combination of 1 and 2 photon processes. Since $400 \mathrm{~nm}$ photons have energy just below the band gap of $\mathrm{ZnO}$ two-photon absorption is expected to be very efficient, and indeed almost saturated. Both one-photon absorption and two-photon absorption are therefore expected to give rise to $\mathrm{THz}$ signals that vary approximately linearly in magnitude with excitation power. This makes it difficult for us to experimentally differentiate onephoton from two-photon absorption. For 2-photon dominated absorption, the decay of excitation power $P$ in the propagation direction $z$ would be described by $d P / d z=-\beta^{(2)} P^{2}$, with the 2-photon absorption coefficient estimated by extrapolating Fig.1 in Ref. 36 to be $\beta^{(2)} \sim 0.04 \mathrm{mM} \mathrm{W}^{-1}$. The $400 \mathrm{~nm}$ power is estimated by dividing the fluence by the pulse duration $(P$ $\sim 1.3 \mathrm{TW} \mathrm{m}^{-2}$ for $0.2 \mathrm{~J} \mathrm{~m}^{-2}$ in a $150 \mathrm{fs}$ pulse). For 1 -photon absorption in the tail of the band gap, absorption is described by $d P / d z=-\beta^{(1)} P$, with $\beta^{(1)} \sim 1 \times 10^{3} \mathrm{~cm}^{-1}$ (Ref. 37). $l_{0}$ is defined as the distance over which the excitation density (proportional to $P^{2}$ and $P$ for respective cases) decays by $1 / e$, and is $\sim 10 \mu \mathrm{m}$ for both 1-photon and 2-photon absorption of $400 \mathrm{~nm}$. For $266 \mathrm{~nm}$ excitation, 1-photon absorption dominates $\left[\beta^{(1)} \sim 2\right.$ $\times 10^{5} \mathrm{~cm}^{-1}$ (Ref. 37)], giving rise to $l_{0} \sim 50 \mathrm{~nm}$. It should be noted that the conductivity extraction analysis neglects effects of charge diffusion in between excitation and the arrival of the $\mathrm{THz}$ probe, so that the calculated $l_{0}$ is a lower limit, and the conductivities derived here are upper limits.

${ }^{15}$ A. Nahata, A. S. Weling, and T. F. Heinz, Appl. Phys. Lett. 69, 2321 (1996).

${ }^{16}$ H. K. Nienhuys and V. Sundstrom, Appl. Phys. Lett. 87, 012101 (2005).

${ }^{17} \mathrm{~A}$ crude estimate of the excitation density in the sample can be made from the incident pump fluence $f_{l}$, taking into account reflective losses at the sample interface (with optical dielectric constant $\varepsilon_{\text {opt }}$ taken from Ref. 36) through $N_{0} \approx f_{l} / l_{0} \times \mid[2 /(1$ $\left.\left.+\sqrt{\varepsilon_{\text {opt }}}\right)\right]^{2} \mid$.

${ }^{18}$ E. Mollwo, O. Madelung, M. Schulz, and H. Weiss, in Semiconductors: Physics of II-VI and I-VII Compounds (LandoltBornstein New Series, Springer, Berlin, 1982), Vol. 17, p. 35.

${ }^{19}$ E. Hendry, F. Wang, J. Shan, T. F. Heinz, and M. Bonn, Phys. Rev. B 69, 081101(R) (2004).

${ }^{20}$ J. Shan, F. Wang, E. Knoesel, M. Bonn, and T. F. Heinz, Phys. Rev. Lett. 90, 247401 (2003).

${ }^{21}$ Wannier excitons have hydrogen-like solutions to the Schrödinger equation. The contribution to the polarizability associated with $1 s$ to $n p$ transitions is given by $\alpha_{n p}(\nu)$ $=\left(\frac{2}{3} \frac{1}{4 \pi \varepsilon_{0}} \frac{|\langle 1 s|e r| n p\rangle|^{2}}{E_{n p}-E_{1 s}}\right) \frac{\nu_{n}^{2}}{\nu_{n}^{2}-\nu^{2}-i \nu\left(\Gamma_{n} / 2 \pi\right)}$, where $\langle 1 s|$ and $|n p\rangle$ are the lowest energy and excited state hydrogenic wave functions, respectively, $\Gamma_{n}$ the absorption linewidth, and $\nu_{n}=\left(E_{n p}-E_{1 s}\right) / h$ the transition resonance frequency determined by the hydrogenic energy levels $E_{n p}$ and $E_{1 s}$ (e.g., $\nu_{2}=8 \mathrm{THz}$ for the $1 s \rightarrow 2 p$ transition). The first part of this equation calculates the static (dc) transition dipole, while the second part allows for a Lorentzian resonance line shape. The lines in Fig. 2(c) are calculated using the Clausius-Mossotti equation to relate polarizability to conductivity (see Ref. 29), summing over all $p$ states, assuming a linewidth $\Gamma_{n}=6 \mathrm{THz}$ (implying a sub-picosecond lifetime for excited states) and exciton density $N_{\text {exc }}=2.6 \times 10^{22} \mathrm{~m}^{-3}$. At THz frequencies the exciton response is dominated by the dc polar- 
izability $\sim 80000 \AA^{3}$, giving rise to the predominantly imaginary conductivity observed in Fig. 5.2(c). The $1 s \rightarrow 2 p$ resonance at $\nu_{2}=8 \mathrm{THz}$, with a linewidth $\Gamma_{2}=6 \mathrm{THz}$ to describe energy level broadening, accounts for the observed small real contribution to the conductivity.

${ }^{22}$ D. C. Reynalds et al., J. Appl. Phys. 88, 2152 (2000).

${ }^{23}$ S. B. Zhang, S. H. Wei, and A. Zunger, Phys. Rev. B 63, 075205 (2001).

${ }^{24}$ T. Koida et al., Appl. Phys. Lett. 82, 532 (2003).

${ }^{25}$ J. R. Goldman and J. A. Prybyla, Phys. Rev. Lett. 72, 1364 (1994).

${ }^{26}$ F. E. Low and D. Pines, Phys. Rev. 98, 414 (1955).

${ }^{27}$ J. Bardeen and W. Shockley, Phys. Rev. 80, 72 (1950).

${ }^{28}$ In equilibrium, and assuming geminate recombination, the dissociation and recombination rates, $k_{\mathrm{dis}}$ and $k_{\mathrm{rec}}$, are related through $k_{\text {dis }} N_{\text {exc }}=k_{\text {rec }} N_{\text {free }}$, where $N_{\text {exc }}$ is the exciton density and $N_{\text {free }}$ the free electron density. The temperature dependent dissociated fraction, as defined in text, is given by $N_{\text {free }} /\left(N_{\text {free }}+N_{\text {exc }}\right)$ $=1 /\left(1+k_{\mathrm{rec}} / k_{\mathrm{dis}}\right)=1 /\left(1+A e^{E / k T}\right)$, where $E$ is the exciton binding energy and $A$ describes the ratio of recombination and dissocia- tion rates. The best fit to the data in Fig. 4(c) is for $E$ $=60 \mathrm{meV}$ and $A=0.02$. Also shown are the fits for $E=30 \mathrm{meV}$ (dotted line) and $E=120 \mathrm{meV}$ (dashed line). In this simple model, we assume the parameter $A$ to be independent of density, though on longer time scales one might expect some nongeminate recombination to occur.

${ }^{29}$ E. Hendry, Ph.D. thesis, University of Amsterdam, 2005.

${ }^{30}$ V. I. Klimov et al., Science 287, 1011 (2000).

${ }^{31}$ Several effects, including limited temporal resolution, local heating, diffusion, and inhomogeneous excitation, lead to measured dynamics which are not true second order.

${ }^{32}$ K. G. Svantesson et al., J. Phys. C 12, 5111 (1979).

${ }^{33}$ T. J. Inagaki and M. Aihara, Phys. Rev. B 65, 205204 (2002).

${ }^{34}$ R. Huber, R. A. Kaindl, B. A. Schmid, and D. S. Chemla, Phys. Rev. B 72, 161314(R) (2005).

${ }^{35}$ H. Deng et al., Proc. Natl. Acad. Sci. U.S.A. 100, 15318 (2003).

${ }^{36}$ I. M. Catalano, A. Cingolani and M. Lepore, Phys. Rev. B 33, 7270 (1986).

${ }^{37}$ G. E. Jellison and L. A. Boatner, Phys. Rev. B 58, 3586 (1998). 